\title{
Hommage à Michel Hery
}

\author{
A tribute to Michel Hery
}

\author{
J. Gligorov $\cdot$ S. Culine $\cdot$ I. Krakowski $\cdot$ P. Marti $\cdot$ J-P Metges $\cdot$ D. Serin $\cdot$ M. Schneider $\cdot$ M. Spielmann \\ (C) Springer-Verlag France 2014
}

Que l'on parle du «cancérologue du rocher » et tout le monde le reconnaîtra. Michel Hery n'est plus, notre ami nous a quittés.

Oncologue-Radiothérapeute, il a vécu son métier comme sa vie, avec passion. Enthousiaste dans tout ce qu'il entreprenait, il a attiré et mobilisé autour de lui, dans toutes ses manifestations, non seulement la plupart des oncologues français, mais aussi francophones ainsi que de nombreux experts internationaux.

Michel Hery avait débuté sa carrière de cancérologue à Paris où il avait été notamment formé à l'institut Gustave Roussy auprès de Maurice Tubiana avec qui il avait gardé jusqu'au bout des rapports d'affection respectueuse. Après des fonctions de médecin radiothérapeute à l'IGR, il avait été attiré par le large et cette mer Méditerranée qui l'avait adopté, lui le Breton, plus du Sud que les autochtones euxmêmes.

\section{J. Gligorov $(\square)$}

APHP Tenon, F-75020 Paris, France

e-mail : joseph.gligorov@tnn.aphp.fr

S. Culine

Hôpital Saint-Louis, F-75010 Paris, Faculté Paris 7, France

I. Krakowski

Institut de Cancérologie de Lorraine - Alexis Vautrin,

F-54519 Vandœuvre lès Nancy, France

P. Marti

Centre Hospitalier de la Côte Basque, F-64100 Bayonne, France

J-P Metges

CHU Brest, Hôpital Morvan, F-29609 Brest cedex, France

D. Serin

Institut Sainte-Catherine, F-84086 Avignon cedex 02, France

M. Schneider

Centre Antoine-Lacassagne, F-06189 Nice cedex 02, France

M. Spielmann

Gustave Roussy, F-94805 Villejuif Cedex, France
Arrivé dans les années 70 à Cannes à la clinique Méridienne, l'amitié qu'il porte à Moïse Namer et l'intérêt pour l'organisation des soins le conduisent à accepter le poste de chef de service en Oncologie-Radiothérapie au centre Antoine Lacassagne, avant de définitivement amarrer sa carrière médicale à quelques kilomètres de là, à Monaco.

Il y aura créé avant tout un service et une équipe répondant aux besoins de la population monégasque. Chef de service de l'Oncologie-Radiothérapie à l'hôpital PrincesseGrace, il aura participé indiscutablement au rayonnement médical de cette ville, non seulement par son implication professionnelle, mais également par son engagement pédagogique en y organisant successivement les Biennales Monégasques de Cancérologie, puis le congrès Monaco Age Oncologie.

Ces manifestations sont devenues très vite un lieu d'échanges à son image, amicaux et emportés de discussions. Nous avons tous été sollicités pour l'élaboration de ces différents programmes de congrès et l'une des priorités essentielles que Michel nous rappelait était de garder le temps pour l'échange et la discussion.

Ces congrès étaient encore une fois à son image, passionnés et animés, mais laissant aussi une large place à l'accueil et à l'amitié. Nous n'allions pas aux Biennales ni au MAO, nous étions accueillis par Michel, chez lui à Monaco.

Nombreux furent également ses engagements ailleurs. Que ce soit pour la création et la poursuite des cours supérieurs francophones de sénologie de St-Paul-de-Vence, ou bien les cours francophones de l'ESO.

Au-delà de son implication professionnelle, Michel avait de nombreuses passions et je n'en citerai que deux : les plaisirs du palais (il aimait partager et découvrir les boissons des dieux) et les chevaux. Finalement, il avait tout des classiques romains : le panache, le plaisir et le goût de l'aventure.

Et puisque « l'air du paradis est celui qui souffle entre les oreilles d'un cheval » comme le dit un proverbe arabe, à toi l'ami, à toi qui les aimais tant tes chevaux qui ont défendu ta casaque bleue et blanche, nous te saluons, mais ne t'oublions pas... Au galop, je l'espère, pour de nouvelles aventures car tu aimais avant tout... la vie. 